Pacific Journal of Mathematic 


\title{
ON THE STRUCTURE OF COMMUTATIVE PERIODIC SEMIGROUPS
}

\author{
B. D. Arendt and C. J. Stuth
}

\begin{abstract}
It is well known that a commutative periodic semigroup is a semilattice of one-idempotent (or unipotent) semigroups. Thus the characterization of commutative periodic semigroups reduces to two subproblems: (1) the structure of commutative periodic unipotent semigroups, and (2) the means for putting these together in the semilattice. In this paper a complete solution is given for problem (1), while problem (2) is solved for the special case where each unipotent subsemigroup is cyclic.
\end{abstract}

If $S$ is a semigroup with zero $\left(S=S^{0}\right)$, then the concepts of nilpotence and the kernel of a homomorphism may be defined in the usual ring-theoretic sense. Thus $x \in S$ is said to be nilpotent if $x^{n}=0$ for some $n$, and the kernel of a homomorphism is the complete inverse image of zero. $S$ is said to be nil if every element of $S$ is nilpotent. Let $T$ be a semigroup with zero and $S$ be any semigroup. Denote by $T^{*}$ the nonzero elements of $T$. A mapping $\alpha$ from $T^{*}$ into $S$ is said to be a partial homomorphism if $a, b \in T^{*}, a b \neq 0$ implies $(a b) \alpha=(a \alpha)(b \alpha)$.

It is easily seen that a commutative semigroup $S$ is periodic and unipotent if and only if $S$ is the ideal extension of a periodic abelian group $G$ by a commutative nil semigroup $T$. Furthermore, every such extension is determined by the partial homomorphisms of $T^{*}$ into $G$ [2, Th. 4.19]. Thus our solution to (1) is obtained by determining the structure of commutative nil semigroups and using the characterization of partial homomorphisms found in [1].

1. Commutative nil semigroups. An element $x$ of a semigroup $S$ is said to be prime if $x$ does not belong to $S^{2}$. $S$ is said to have unique factorization if every nonzero element of $S$ can be written uniquely as a product of powers of primes. Of course, if $S$ is not commutative, we must take the order of the factors into account. The following result is a corollary to Theorem 1 of [1].

LEMMA 1. $S=S^{0}$ is commutative nil if and only if there exists a commutative nil semigroup $U$ with unique factorization and a homomorphism from $U$ onto $S$ with trivial kernel.

If $S=S^{0}$ is commutative and $x \in S$ we define the annihilator of 
$x$ as in ring theory by $\operatorname{Ann}(x)=\{y: x y=0\}$. Define a relation $R$ on $S$ by $0 R 0$, and for $x \neq 0 \neq y, x R y$ if and only if Ann $(x)=\operatorname{Ann}(y)$. It is straightforward to show that $R$ is a congruence on $S$ with trivial kernel (the class containing zero). Moreover, if $\tau$ is any congruence on $S$ with trivial kernel, then $\tau \leqq R$.

THEOREM 2. Let $S$ be a commutative nil semigroup and let $\left\{A_{i}: i \in I\right\}$ be the set of $R$-classes of $S$. Then:

(i ) Any partition $B_{i j}, j \in J_{i}$, of some $R$-class $A_{i}$ induces a congruence $\tau_{i}$ with trivial kernel on $S$ such that the $B_{i j}$ are congruence classes of $\tau_{i}$.

(ii) Every congruence on $S$ with trivial kernel is the intersection of a collection of such congruences $\tau_{i}$.

Proof. Let $\left\{B_{i j}: j \in J_{i}\right\}$ be a partition of some $R$-class $A_{i}$. Define $\tau_{i}$ as follows:

(a) $0 \tau_{i} 0$;

(b) for $x, y \in A_{i}, x \tau_{i} y \Leftrightarrow x, y \in B_{i j}$, for some $j \in J_{i}$;

(c) for $x \neq 0 \neq y$ and $x, y \notin A_{i}, x \tau_{i} y \Leftrightarrow$ for all $z \in S$ and $j \in J_{i}$, $x z \in B_{i j} \Leftrightarrow y z \in B_{i j}$.

$\tau_{i}$ is an equivalence relation on $S$. To show $\tau_{i}$ is a congruence we consider separately the last two cases of the definition.

First, suppose $x, y \notin A_{i}$ and $x \tau_{i} y$. If $x z \in A_{i}$ then $x z \in B_{i j}$ for some $j$ and so $y z \in B_{i j}$ and $(x z) \tau_{i}(y z)$. If $x z \notin A_{i}$, then $y z \notin A_{i}$, and $x(z w) \in B_{i j}$ if and only if $y(z w) \in B_{i j}$ for all $w \in S$, so that $(x z) \tau_{i}(y z)$ for all $z \in S$. Secondly, let $x, y \in A_{i}$ and $x \tau_{i} y$. Then $x, y \in B_{i j}$ for some $j$. Now if $A_{i}=\{0\}$, then $(x z) \tau_{i}(y z)$ follows immediately, so assume $A_{i} \neq\{0\}$. If $x z \in A_{i}$ for some $z \in S$, then $x R(x z)$ which implies $x R\left(x z^{n}\right)$ for all $n$, but $S$ is nil, so $x R 0$ and $x=0$, a contradiction. Thus for $x, y \in A_{i} \neq\{0\}$ we have $x z, y z \notin A_{i}$ for all $z$ in $S$, so trivially $(x z) \tau_{i}(y z)$, and we have proved (i).

To prove (ii), let $\tau$ be any congruence on $S$ with trivial kernel. Then $\tau \leqq R$, and $\tau$ induces a partition $B_{i j}$ on each $R$-class $A_{i}$. If we define $\tau_{i}$ to be the congruence induced on $S$ by each such partition (as in the proof of (i)), then it follows directly that $\tau=\cap\left\{\tau_{i}: i \in I\right\}$ since $\tau$ and $\tau_{i}$ agree on $A_{i}$ for all $i$.

All commutative nil semigroups with unique factorization are easily determined [1]. Let $F$ be the free commutative semigroup on $X$ with ideal $K$. Then $F / K$ is nil if and only if $K$ contains some positive power of each $x$ in $X$. Combining Theorem 2 with Lemma 1 we obtain all commutative nil semigroups.

We remark that there are sufficient congruences in Theorem 2 to separate distinct elements of $S$. 
2. Semilattices of cyclic semigroups. A cyclic semigroup $S$ has an idempotent if and only if it is finite, and for these semigroups the concepts of index and period are defined as in [2, p. 19]. If $S$ is infinite cyclic we may say it has infinite index and zero period, so that these terms are defined for all cyclic semigroups. With that convention in mind the following theorem characterizes semilattices of all cyclic semigroups, not just those with idempotents, and the solution of problem (2) mentioned in the introduction is obtained by assuming each cyclic semigroup is finite.

By $Z, Z^{+}$and $Z_{0}$ we mean the integers, positive integers and nonnegative integers respectively.

TheOREM 3. Let $Y$ be any semilattice and $\left\{S_{\alpha}: \alpha \in Y\right\}$ a collection of disjoint cyclic semigroups where $S_{\alpha}=\left\langle a_{\alpha}\right\rangle$ with index $n_{\alpha}$, and period $p_{\alpha}$. For all $\alpha \geqq \beta \in Y$, choose $f(\alpha, \beta) \in Z_{0}$ such that

(i ) $f(\alpha, \alpha)=1$;

(ii) $n_{\delta \lambda} \neq 1 \Rightarrow f(\lambda, \delta \lambda)+f(\delta, \delta \lambda) \neq 0$.

Define $g: Y \times Y \rightarrow Z$ such that:

(iii) $g(\alpha, \beta)=g(\beta, \alpha)$ for all $\alpha, \beta \in Y$;

(iv) $\alpha \neq \alpha \beta \neq \beta$ and $f(\alpha, \alpha \beta)+f(\beta, \alpha \beta)=0 \Rightarrow g(\alpha, \beta)=1$;

(v) $\alpha \neq \alpha \beta \neq \beta$ and $f(\alpha, \alpha \beta)+f(\beta, \alpha \beta)=n_{\alpha \beta}-1 \Rightarrow g(\alpha, \beta)=1$ or 0 ;

(vi) $\alpha \neq \alpha \beta \neq \beta$ and $f(\alpha, \alpha \beta)+f(\beta, \alpha \beta)=n_{\alpha \beta}-1+k p_{\alpha \beta}+S$ for some $k \in Z^{+}$and $0 \leqq S \leqq p-1 \Rightarrow g(\alpha, \beta)=-k$ or $1-k$;

(vii) $g(\alpha, \beta)=0$ otherwise.

Let $S=\cup\left\{S_{\alpha} \mid \alpha \in Y\right\}$ and define multiplication in $S$ by

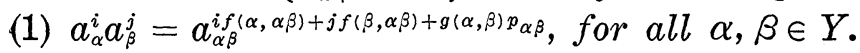

Further assume that $f$ and $g$ are defined such that

(viii) $a_{\alpha}^{i}\left(a_{\beta}^{j} a_{r}^{k}\right)=a_{\beta}^{j}\left(a_{\alpha}^{i} a_{\gamma}^{k}\right)=a_{r}^{k}\left(a_{\alpha}^{i} a_{\beta}^{j}\right)$ for all $\alpha, \beta, \gamma \in Y$, and $i, j, k \in$ $\{1,2\}$ such that $i+j+k \leqq 4$.

Then $S$ is a commutative semigroup. Conversely, every commutative semigroup which is a semilattice of cyclic semigroups may be constructed in this manner.

Proof. Suppose $S=\cup\left\{S_{\alpha}: \alpha \in Y\right\}$ is a commutative semigroup which is a $Y$-semilattice of the $S_{\alpha}$. Denote by $G_{\alpha}$ the maximal subgroup of $S_{\alpha}$, if it exists. For $\alpha>\beta$ in $Y$, define $f(\alpha, \beta)=\exp \left(a_{\alpha} a_{\beta}\right)-1$ where $\exp \left(a_{\alpha} \alpha_{\beta}\right)$ is the least positive integer $t$ such that $a_{\alpha} a_{\beta}=a_{\beta}^{t}$. Define $f(\alpha, \alpha)=1$ for all $\alpha \in Y$.

Now let $\alpha, \beta \in Y$. Then $a_{\alpha} a_{\beta}=a_{\alpha \beta}^{t}$ for some least positive integer t. We have $a_{\alpha \beta}^{t+1}=a_{\alpha}\left(a_{\beta} a_{\alpha \beta}\right)=a_{\alpha \beta}^{f(\alpha, \alpha \beta)+f(\beta, \alpha \beta)+1}$, so

$$
t=f(\alpha, \alpha \beta)+f(\beta, \alpha \beta)+g(\alpha, \beta) p_{\alpha \beta}
$$


for some integer $g(\alpha, \beta)$. By induction

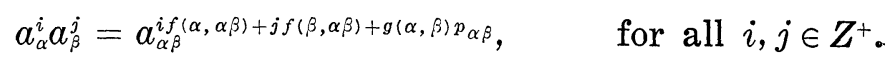

Suppose $\alpha \neq \alpha \beta \neq \beta$ in $Y$ in the following three cases.

Case 1. $f(\alpha, \alpha \beta)+f(\beta, \alpha \beta)=0$ : Then $f(\alpha, \alpha \beta)=f(\beta, \alpha \beta)=0$ so $n_{\alpha \beta}=1$ and $p_{\alpha \beta}=t$. Thus (ii) and (iv) are satisfied because $g(\alpha, \beta)=1$.

Case 2. $f(\alpha, \alpha \beta)+f(\beta, \alpha \beta)=n_{\alpha \beta}-1$ : Then $\left(a_{\alpha \beta}\right)^{n}{ }_{\alpha \beta}=a_{\alpha \beta}^{t+1}$. If $a_{\alpha} a_{\beta} \notin G_{\alpha \beta}$, then $t=n_{\alpha \beta}-1$ and $g(\alpha, \beta)=0$. On the other hand if $a_{\alpha} a_{\beta} \in G_{\alpha \beta}$, then $t=p_{\alpha \beta}+n_{\alpha \beta}-1$ so that $g(\alpha, \beta)=1$. Thus (v) holds.

Case 3. $f(\alpha, \alpha \beta)+f(\beta, \alpha \beta)=n_{\alpha \beta}-1+k p_{\alpha \beta}+S$ where $k \in Z^{+}$, $0 \leqq S \leqq p-1$ : If $a_{\alpha} a_{\beta} \notin G_{\alpha \beta}$, then $t=n_{\alpha \beta}-1, S=0$, and $g(\alpha, \beta)=-k$. If $a_{\alpha} \alpha_{\beta} \in G_{\alpha \beta}$ and $S \neq 0$, then $t=n_{\alpha \beta}-1+S$ and $g(\alpha, \beta)=-k$. If $a_{\alpha} a_{\beta} \in G_{\alpha \beta}$ and $S=0$, then $t=n_{\alpha \beta}-1+p$ and $g(\alpha, \beta)=1-k$.

In every other situation we see $a_{\alpha \beta}^{t}=a_{\alpha \beta}^{f(\alpha, \alpha \beta)+f(\beta, \alpha \beta)}$ where the exponents are in fact equal, so that $g(\alpha, \beta)=0$, giving (vii).

Now that $g(\alpha, \beta)$ is defined for all $\alpha, \beta \in Y$ it is clear that (iii) is satisfied, and (viii) is obvious by the associativity of $S$.

Conversely, suppose $S=\cup\left\{S_{\alpha}: \alpha \in Y\right\}$ and the functions $f$ and $g$ are defined satisfying (i)-(viii) with multiplication given by (1). Multiplication is commutative by (1) and (iii). For $\alpha>\beta>\gamma$ in $Y$, condition (viii) implies

$$
\begin{aligned}
& f(\alpha, \gamma) \equiv f(\alpha, \beta) f(\beta, \gamma) \bmod p_{\gamma} \text { or } \\
& f(\alpha, \gamma)=f(\alpha, \beta) f(\beta, \gamma) .
\end{aligned}
$$

Let $a_{\alpha}^{i}, a_{\beta}^{j}, a_{\gamma}^{k}$ be any three elements of $S$. Then $a_{\alpha}^{i}\left(a_{\beta}^{j} a_{\gamma}^{k}\right), a_{\beta}^{j}\left(a_{\alpha}^{i} a_{\gamma}^{k}\right)$ and $a_{\gamma}^{k}\left(a_{\alpha}^{i} a_{\beta}^{j}\right)$ are powers of $a_{\alpha \beta \gamma}$ (using (1)) with exponents which we denote $e_{1}, e_{2}$ and $e_{3}$ respectively. If $\min \left\{e_{1}, e_{2}, e_{3}\right\} \geqq n_{\alpha \beta \gamma}$, then the associativity follows by applying (2) to corresponding parts of the exponents $e_{1}, e_{2}$ and $e_{3}$. So we may assume that, say, $e_{1}<n_{\alpha \beta}$. Then from (viii) it follows that the exponents of $a_{\alpha}\left(a_{\beta} a_{\gamma}\right), a_{\beta}\left(a_{\alpha} a_{\gamma}\right)$ and $a_{\gamma}\left(a_{\alpha} a_{\beta}\right)$, which we denote by $r_{1}, r_{2}$ and $r_{3}$ respectively, are equal. If $i \geqq 2$, then $a_{\alpha}^{2}\left(a_{\beta} a_{\gamma}\right)=a_{\beta}\left(a_{\alpha}^{2} a_{\gamma}\right)=a_{\gamma}\left(a_{\alpha}^{2} a_{\beta}\right) \notin G_{\alpha \beta \gamma}$ so that the exponents of these expressions are equal, and in conjunction with the previous statement we get

$$
f(\alpha, \alpha \beta \gamma)=f(\alpha, \alpha \beta) f(\alpha \beta, \alpha \beta \gamma)=f(\alpha, \alpha \gamma) f(\alpha \gamma, \alpha \beta \gamma) .
$$

Similarly, if $j \geqq 2$ or $k \geqq 2$, then we have the respective equations

$$
f(\beta, \alpha \beta \gamma)=f(\beta, \alpha \beta) f(\alpha \beta, \alpha \beta \gamma)=f(\beta, \beta \gamma) f(\beta \gamma, \alpha \beta \gamma)
$$




$$
f(\gamma, \alpha \beta \gamma)=f(\gamma, \alpha \gamma) f(\alpha \gamma, \alpha \beta \gamma)=f(\gamma, \beta \gamma) f(\beta \gamma, \alpha \beta \gamma) .
$$

Adding $(i-1) f(\alpha, \alpha \beta \gamma)+(j-1) f(\beta, \alpha \beta \gamma)+(k-1) f(\gamma, \alpha \beta \gamma)$ to each member of the equation $r_{1}=r_{2}=r_{3}$, and using (3), (4) and (5) when appropriate, we obtain $e_{1}=e_{2}=e_{3}$. Therefore $S$ is associative.

For the special case when each $S_{\alpha}$ is infinite cyclic, Theorem 3 is greatly simplified, and we state that result in the following corollary.

COROLLARY 4. Let $Y$ be any semilattice and $\left\{S_{\alpha}=\left\langle a_{\alpha}\right\rangle: \alpha \in Y\right\}$ a collection of disjoint infinite cyclic semigroups indexed by $Y$. For all $\alpha \geqq \beta$ in $Y$ choose $f(\alpha, \beta) \in Z_{0}$ such that

(i) $f(\alpha, \alpha)=1$

(ii) $f(\lambda, \delta \lambda)+f(\delta, \lambda \delta) \neq 0, \quad \lambda, \delta \in Y$.

(iii) $\alpha \geqq \beta \geqq \gamma \Rightarrow f(\alpha, \gamma)=f(\alpha, \beta) f(\beta, \gamma)$. Lst $S=\cup\left\{S_{\alpha}: \alpha \in Y\right\}$ and define multiplication in $S$ by

$$
a_{\alpha}^{i} a_{\beta}^{j}=a_{\alpha \beta}^{i f(\alpha, \alpha \beta)+j f(\beta, \alpha \beta)}, \quad \alpha, \beta \in Y .
$$

Then $S$ is a commutative semigroup. Conversely, every commutative semigroup which is a semilattice of infinite cyclic semigroups is determined in this manner.

Proof. It is easily verified that (iii) is sufficient for the equality of the three exponents that arise from the product $a_{\alpha}^{i} \alpha_{\beta}^{j} a_{\gamma}^{k}$. Conversely, if $S$ is associative and $\alpha \geqq \beta \geqq \gamma$ then the exponents from $a_{\alpha}\left(a_{\beta} a_{\gamma}\right)=$ $a_{r}\left(a_{\alpha} a_{\beta}\right)$ will give (iii).

We remark that condition (viii) of Theorem 3 says essentially that associativity of third degree and fourth degree terms is sufficient to guarantee all associativity. We conclude with an example to show that in this respect Theorem 3 is the best possible result.

Let $Y$ be the semilattice consisting of $\alpha, \beta, \gamma=\alpha \gamma, \alpha \beta$, and $\alpha \beta \gamma=\beta \gamma$. Let the cyclic semigroups indexed by $Y$ be chosen such that $n_{\beta \gamma}=11, p_{\alpha \beta}=p_{\beta \gamma}=1$ and $n_{\alpha \beta}=2$. Define $f$ and $g$ by $f(\alpha, \gamma)=1$, $f(\alpha, \alpha \beta)=f(\alpha, \beta \gamma)=f(\gamma, \beta \gamma)=0, f(\beta, \alpha \beta)=2, f(\beta, \beta \gamma)=f(\alpha \beta, \beta \gamma)=11$, and $g(\alpha, \beta)=g(\beta, \gamma)=g(\gamma, \alpha \beta)=-1$. Conditions (i)-(vii) of Theorem 3 are satisfied. It can be shown that any term of the form $x y z$ where $x, y, z$ are first powers of the generators is associative. In fact, any term of the form $a_{\alpha}^{i} a_{\beta} a_{\gamma}^{k}$ is associative. However $\left(a_{\alpha} a_{\beta}^{2}\right) a_{r}=a_{\alpha}\left(a_{\beta}^{2} a_{\gamma}\right)$ so that the union of these is not a semigroup under the multiplication (1). 


\section{BIBLIOGRAPHY}

1. B. D. Arendt and C. J. Stuth, On partial homomorphism of semigroups, Pacific J. Math., 35 (1970).

2. A. H. Clifford and G. B. Preston, Algebraic theory of semigroups, Vol. 1, Math. Surveys No. 7, Amer. Math. Soc., Providence, R. I., 1961.

Received October 7, 1969. The first author was partially supported by University of Missouri Research Grant. The second author was partially supported by NSF Grant No. 9618.

The University of Missouri

Columbia, Missouri 


\section{PACIFIC JOURNAL OF MATHEMATICS}

\section{EDITORS}

H. SAmelson

Stanford University

Stanford, California 94305

RICHARd PieRCe

University of Washington

Seattle, Washington 98105

\author{
J. DugundJI \\ Department of Mathematics \\ University of Southern California \\ Los Angeles, California 90007 \\ RICHARD ARENS \\ University of California \\ Los Angeles, California 90024
}

ASSOCIATE EDITORS
E. F. BECKENBACH
B. H. NeumanN
F. WOLF
K. YosHIDA

\section{SUPPORTING INSTITUTIONS}

UNIVERSITY OF BRITISH COLUMBIA

CALIFORNIA INSTITUTE OF TECHNOLOGY

UNIVERSITY OF CALIFORNIA

MONTANA STATE UNIVERSITY

UNIVERSITY OF NEVADA

NEW MEXICO STATE UNIVERSITY

OREGON STATE UNIVERSITY

UNIVERSITY OF OREGON

OSAKA UNIVERSITY

UNIVERSITY OF SOUTHERN CALIFORNIA
STANFORD UNIVERSITY

UNIVERSITY OF TOKYO

UNIVERSITY OF UTAH

WASHINGTON STATE UNIVERSITY

UNIVERSITY OF WASHINGTON

AMERICAN MATHEMATICAL SOCIETY CHEVRON RESEARCH CORPORATION TRW SYSTEMS

NAVAL WEAPONS CENTER 


\section{Pacific Journal of Mathematics}

\section{Vol. 35, No. $1 \quad$ September, 1970}

B. D. Arendt and C. J. Stuth, On the structure of commutative periodic semigroups ..................................... 1

B. D. Arendt and C. J. Stuth, On partial homomorphisms of semigroups .... 7

Leonard Asimow, Extensions of continuous affine functions ............ 11

Claude Elias Billigheimer, Regular boundary problems for a five-term recurrence relation ................................... 23

Edwin Ogilvie Buchman and F. A. Valentine, A characterization of the

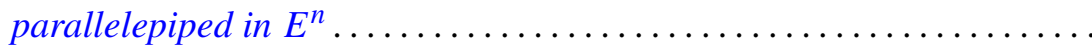
53

Victor P. Camillo, A note on commutative injective rings ............ 59

Larry Jean Cummings, Decomposable symmetric tensors ............. 65

J. E. H. Elliott, On matrices with a restricted number of diagonal values ... 79

Garth Ian Gaudry, Bad behavior and inclusion results for multipliers of type

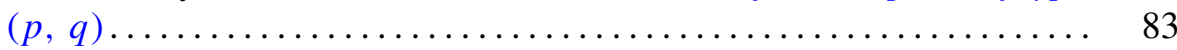

Frances F. Gulick, Derivations and actions .................. 95

Langdon Frank Harris, On subgroups of prime power index . . . . . . . . 117

Jutta Hausen, The hypo residuum of the automorphism group of an abelian

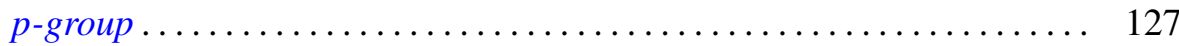

R. Hrycay, Noncontinuous multifuctions . ................... 141

A. Jeanne LaDuke, On a certain generalization of $p$ spaces ............ 155

Marion-Josephine Lim, Rank preservers of skew-symmetric matrices...... 169

John Hathway Lindsey, II, On a six dimensional projective representation of the Hall-Janko group ................................... 175

Roger McCann, Transversally perturbed planar dynamical systems . . . . . 187

Theodore Windle Palmer, Real $C^{*}$-algebras ................... 195

Don David Porter, Symplectic bordism, Stiefel-Whitney numbers, and a

Novikov resolution ....................................... 205

Tilak Raj Prabhakar, On a set of polynomials suggested by Laguerre

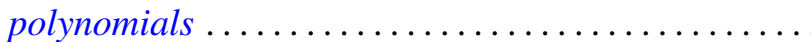

B. L. S. Prakasa Rao, Infinitely divisible characteristic functionals on locally convex topological vector spaces...................

John Robert Reay, Caratheodory theorems in convex product structures ...

Allan M. Sinclair, Eigenvalues in the boundary of the numerical range. .

David R. Stone, Torsion-free and divisible modules over matrix rings ....

William Jennings Wickless, A characterization of the nil radical of a

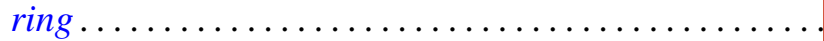

